\title{
Qualidade da relação da gestante com as pessoas próximas e o aleitamento materno
}

\author{
Pregnant relationship quality with the closest people and breastfeeding
}

Calidad de la relación de la embarazada con las personas cercanas y la lactancia materna

\author{
Janaine Fragnan Peres ${ }^{1}$ (1) \\ Ariana Rodrigues Silva Carvalho² (1) \\ Cláudia Silveira Viera ${ }^{2,3}$ (1) \\ Ana Maria Linares ${ }^{4}$ (1) \\ Marialda Moreira Christoffel ${ }^{5,6}$ (1) \\ Beatriz Rosana Gonçalves de Oliveira
}

Toso $^{2,3}$ (1)

1. Universidade Estadual do Oeste do Paraná. Cascavel, PR, Brasil.

2. Universidade Estadual do Oeste do Paraná, Departamento de Enfermagem. Cascavel, PR, Brasil.

3. Universidade Estadual do Oeste do Paraná, Programa de Pós-Graduação em Biociências e Saúde. Cascavel, PR, Brasil.

4. University of Kentucky, College of Nursing. Lexington, KY, Estados Unidos da América.

5. Universidade Federal do Rio de Janeiro, Escola de Enfermagem Anna Nery, Programa de Pós-graduação em Enfermagem. Rio de Janeiro, RJ, Brasil.

6. Universidade Federal do Rio de Janeiro, Departamento de Enfermagem. Macaé, RJ, Brasil.

Autor correspondente:

Janaine Fragnan Peres.

E-mail: janainefp@hotmail.com.

Recebido em 20/05/2020.

Aprovado em 22/09/2020.

DOl:https://doi.org/10.1590/2177-9465-EAN-2020-0163

\section{RESUMO}

Objetivos: Identificar a pessoa próxima à gestante, que atua como sua fonte de apoio primária, bem como avaliar a qualidade dessa relação por meio do instrumento qualidade da relação com as pessoas próximas e sua influência no aleitamento materno. Método: Estudo quantitativo, descritivo e exploratório realizado com uma amostra não probabilística consecutiva de 152 gestantes, em Unidades de Saúde de município de médio porte na região Oeste do Paraná, durante o ano de 2019, para responder à pergunta de pesquisa "a qualidade da relação da mulher na gestação com a sua fonte de apoio primária tem implicações na amamentação? Utilizou-se, para obtenção dos dados, a escala "Qualidade da relação com as pessoas próximas-ARl", que classifica o vínculo por meio de pontos que podem variar de 40 a 128, sendo que quanto maior, também maior será a qualidade do vínculo com aquela pessoa. Os dados foram analisados por estatística descritiva. Resultados: As mulheres identificaram o companheiro/esposo $(58,6 \%)$ e outros membros da família $(40,1 \%)$ como as pessoas mais importantes e atuantes como sua fonte de apoio primária. A média do escore foi de 103,5, apontando que o relacionamento é saudável e predominam aspectos positivos. Conclusão: Quanto mais positivos os resultados, maior é o suporte recebido pela gestante e, consequentemente, maior a influência para que a mulher inicie e mantenha o aleitamento materno exclusivo. Implicações para a prática clínica: A escala utilizada pode ser aplicada com regularidade na atenção primária para o enfermeiro identificar vínculos frágeis que influenciariam a amamentação.

Palavras-chave: Aleitamento materno; Apoio social; Gestantes; Saúde da Mulher; Atenção Primária à Saúde.

\section{ABSTRACT}

Objectives: To identify the closest person to the pregnant woman, who acts as her primary support source, and evaluate the relationship quality through the instrument quality with the persons close to her and their influence on breastfeeding. Method: Quantitative, descriptive, and exploratory study with a consecutive non-probabilistic sample of 152 pregnant women, in Health Units in a medium-sized municipality in the western region of Paraná, during the year 2019, to answer the research question "does the quality of the relationship between women in pregnancy and their primary source of support have implications for breastfeeding?. The "Quality of the relationship with the closest people scale - ARI" was used to obtain the data, which classifies the bond using points that can vary from 40 to 128 , and the higher, the higher will be the quality of the bond with that person. The data were analyzed using descriptive statistics. Results: Women identified the partner/husband (58.6\%) and other family members $(40.1 \%)$ as the most important and active people as their primary support source. The average score was 103.5, indicating that the relationship is healthy and positive aspects predominate. Conclusion: The more positive the results, the greater the support received by the pregnant woman and, consequently, the greater the influence for the woman to initiate and maintain exclusive breastfeeding. Implications for the clinical practice: The scale used can be applied regularly in primary care for nurses to identify fragile bonds that would influence breastfeeding.

Keywords: Breastfeeding; Social Support; Pregnant Women; Women Health; Primary Health Care.

\section{RESUMEN}

Objetivos: Identificar a la persona cercana a la mujer embarazada, que actúa como su principal fuente de apoyo, así como evaluar la calidad de esta relación por medio del instrumento cualidad de la relación con las personas cercanas a ella y su influencia en la lactancia materna. Método: Estudio cuantitativo, descriptivo y exploratorio, realizado con una muestra consecutiva no probabilística de 152 gestantes, en Unidades de Salud de un municipio mediano de la región oeste de Paraná, durante el año 2019, para responder a la pregunta de investigación "¿la calidad de la relación de las mujeres en gestación con su fuente de apoyo principal tiene implicaciones para la lactancia materna? La escala "Calidad de la relación con personas cercanas - ARl" se utilizó para obtener los datos, que clasifican el vínculo mediante puntos que pueden variar de 40 a 128, cuanto mayor sea la calidad, mayor el vínculo con esa persona. Los datos se analizaron mediante estadística descriptiva. Resultados: Las mujeres identificaron a la pareja / esposo $(58.6 \%)$ y otros miembros de la familia $(40.1 \%)$ como las personas más importantes y activas como su principal fuente de apoyo. El puntaje promedio fue de 103.5, lo que indica que la relación es saludable y predominan los aspectos positivos. Conclusión: Cuanto más positivos sean los resultados, mayor será el apoyo recibido por la mujer embarazada y, en consecuencia, mayor será la influencia para que la mujer inicie y mantenga la lactancia materna exclusiva. Implicaciones para la práctica clínica: La escala utilizada puede aplicarse regularmente en la atención primaria para que las enfermeras identifiquen los vínculos frágiles que influirían en la lactancia materna.

Palabras clave: Lactancia Materna; Apoyo social; Mujeres Embarazadas; Salud de la Mujer; Atencion Primaria de Salud. 


\section{INTRODUÇÃO}

O Aleitamento Materno (AM) não se configura apenas em uma prática de nutrição da criança, mais do que isso, envolve processos que estreitam o vínculo entre mãe e filho e trazem benefícios significativos para ambos. Assim como, melhora a qualidade de vida das famílias, pois reduz a incidência de doenças e todas as suas implicações. ${ }^{1}$ Além disso, promove incrementos de ordem econômica para um país, seja por meio do aumento do quociente de inteligência (QI) da criança, seja pela redução nos custos com assistência à saúde e/ou por possuir sustentabilidade ambiental e não causar poluição, desperdício ou uso de embalagens desnecessárias. ${ }^{2}$

Entretanto, muitos são os determinantes que atuam no contexto da amamentação e que precisam ser considerados e avaliados para se obter sucesso nessa prática, a saber: fatores biológicos, econômicos, sociais, culturais e psicológicos. ${ }^{3}$

Nesta conjuntura, tem-se a atuação das redes de apoio social, composta de atores próximos a mulher, ou seja, fontes de apoio primário que lhe fornecem suporte, podendo influenciar positivamente ou negativamente em sua decisão de amamentar e, portanto, devem ser considerados como determinantes na adesão e manutenção da amamentação. ${ }^{4}$

O apoio social é considerado uma ferramenta de suporte psicológico e emocional para as mulheres durante a gestação e, posteriormente, no puerpério. ${ }^{5}$ Pode ser apresentado como apoio emocional, material e financeiro e envolve, ainda, a ajuda nos afazeres domésticos, nos cuidados com o bebê e com os outros filhos. ${ }^{6}$

Nesta vertente, diversos estudos investigam a rede de apoio social de gestantes e puérperas que pode influenciar a prática da amamentação, como constatado por uma pesquisa que o meio familiar e pessoas do contexto comunitário, principalmente as mulheres, são as principais fontes de apoio dessa rede; ${ }^{4}$ e que os conselhos fornecidos pelo marido/companheiro e pela mãe representam importante suporte e ajudam a lidar com dificuldades e desconfortos que podem surgir, reforçando a prática do AM. ${ }^{7}$ Também demonstraram que pessoas que não são familiares, mas que convivem com a mulher, como amigos, vizinhos, pai da criança que não coabita com ela, colegas de trabalho, entre outros, atuam como atores sociais na rede, principalmente para àquelas que não possuem familiares próximos. ${ }^{6}$

Entretanto, a partir do contexto acima descrito, verifica-se que, dentre os estudos com a finalidade de identificar a rede de apoio social da mulher na gestação e sua relação com a prática da amamentação, poucos avaliam e/ou discutem a importância da qualidade desse relacionamento e as ações dos profissionais de saúde necessárias para seu fortalecimento em relação à prática da amamentação. Destaca-se que nessa nova fase de ser mãe, a progenitora muitas vezes se sente incompetente e incapaz para o ato de criação do filho e, na maioria das vezes, não possui o suporte emocional ou físico das pessoas que estão ao seu redor. Pelo contrário, o que ela recebe, frequentemente, são opiniões que a desacreditam como mulher e como mãe. ${ }^{8}$

Assim sendo, o estudo ampara-se na seguinte questão norteadora: A qualidade da relação da mulher na gestação com a sua fonte de apoio primária tem implicações na amamentação?
Com o propósito de responder tal questionamento, objetivou-se identificar a pessoa próxima à gestante, que atua como sua fonte de apoio primária, bem como avaliar a qualidade dessa relação por meio do instrumento qualidade da relação com as pessoas próximas e sua influência no aleitamento materno.

\section{MÉTODO}

O estudo integra um projeto multicêntrico, denominado 'Aleitamento materno exclusivo: determinantes socioculturais no Brasil', sob coordenação da Escola de Enfermagem Anna Nery, da Universidade Federal do Rio de Janeiro (EEAN-UFRJ) que, por sua vez, faz parte de pesquisa internacional sobre o AM nas Américas, denominado 'Lactância materna exclusiva: determinantes socioculturales en Latino América.

Trata-se de estudo quantitativo, descritivo e exploratório realizado com uma amostra não probabilística consecutiva de 152 gestantes, frequentadoras de Unidades de Saúde em um município de médio porte na região Oeste do Paraná, por ocasião da consulta de pré-natal e com seguimento até os seis meses de vida do bebê. A escolha das unidades de saúde, nas quais se realizou a coleta de dados junto às gestantes, deu-se a partir de relatório do setor de epidemiologia do município, identificando-se aquelas unidades com maior número de gestantes cadastradas no ano de 2018. Os critérios de inclusão da amostra foram: ser gestante com idade gestacional entre 30 e 37 semanas, com risco habitual e/ou intermediário (registrado no prontuário e/ou no cartão da gestante), brasileiras e com idade igual ou maior a 18 anos.

Os dados foram coletados pela equipe de pesquisa, composta de mestrandos e alunos de iniciação científica de graduação em Enfermagem, durante o ano de 2019, por meio da escala "Qualidade da relação com as pessoas próximas", traduzida e validada para o português do Brasil pela equipe de pesquisa da EEAN-UFRJ, ${ }^{9}$ a partir da escala em espanhol Calidad de la relación com su persona cercana. ${ }^{10}$ Originalmente, essa escala foi desenvolvida por Hall (1983), na versão inglesa Autonomy and Relatedness Inventory $(A R I) .{ }^{10}$ Tanto nas versões em espanhol quanto em português, a sigla da escala na língua inglesa (ARI) foi mantida.

A escala original (em inglês) foi composta por itens pertencentes ao Marital Autonomy and Relatedness Inventory (MARI), construída por Shaefer e Edgerton (1979) e avalia a qualidade das relações conjugais; e, a partir daí, Hall (1983) adicionou oito itens para avaliar o suporte e a escuta, medida esta baseada em definições precoces de apoio social e conceituações de relações interpessoais, bem como no reconhecimento da necessidade de avaliar o lado não favorável dos relacionamentos próximos. ${ }^{10}$

Assim, o Autonomy and Relatedness Inventory ( $A R I)$ é uma escala de 32 itens, divididos em oito subescalas: Aceitação, Parentesco, Suporte, Escuta, Autonomia, Controle, Controle Hostil e Desapego/Rejeição. Estas, por sua vez, estão agrupadas numa estrutura de fator bidimensional, sendo: Apoio/Atitude Positiva com 20 itens, considerada positiva e Dominio/Controle, com 12 itens, considerada negativa. ${ }^{10}$

A escala adaptada, tanto na versão espanhola (Autonomy and Relatedness Inventory - Spanish version), quanto na brasileira (Qualidade da relação com as pessoas próximas) 
manteve-se com 32 itens e produziu a mesma estrutura fatorial que a ARI - English, com duas dimensões: uma positiva e outra negativa. ${ }^{9} 10$ A dimensão Apoio/Atitude positiva é representada pelos itens $1,3,4,5,7,9,11,13,15,16,17,19,21,23,24,25$, 28, 29, 30 e 31; e Domínio/Controle é representada pelos itens $2,6,8,10,12,14,18,20,22,26,27$ e 32 . Na versão brasileira, a escala apresentou índice de validação de conteúdo de 0,92e o alpha de Cronbach $0,869 .{ }^{9}$

Para a coleta de dados, primeiramente, realizou-se contato telefônico com as coordenadoras das unidades de saúde selecionadas para o estudo, para obtenção das datas e horários da agenda da unidade para atendimento às gestantes, uma vez que a abordagem ocorreu concomitante ao comparecimento destas para atendimento.

Realizou-se o convite para participação no estudo àquelas gestantes que se enquadravam nos critérios de inclusão da pesquisa, antes ou após sua consulta médica e/ou de enfermagem. As gestantes que aceitaram participar da pesquisa foram encaminhadas, de forma individual, a uma sala disponibilizada pela unidade de saúde, com a finalidade de se manter a privacidade, momento em que foi-lhes explicada detalhadamente a pesquisa e seus objetivos, bem como a solicitação, após expressarem concordância, de que assinassem o Termo de Consentimento Livre e Esclarecido - TCLE, para posterior coleta de dados da pesquisa.

$\mathrm{Na}$ coleta de dados, primeiramente, aplicou-se o formulário de caracterização sociodemográfica das participantes e, em seguida, solicitou-se que identificassem a pessoa mais importante de sua vida, de quem se sentiam mais próximas. $\mathrm{Na}$ sequência, foram convidadas a descrever sua percepção do comportamento íntimo em relação a cada item da escala, baseando-se em escala Likert de cinco pontos, variando de (1) "não, nunca" a (5) "muitíssimo, sempre". Para a análise, os valores dos itens foram recodificados de 1 (zero), $2(1,0), 3(2,0)$, $4(3,0), 5(4,0)$. Para calcular a pontuação total, somaram-se os valores correspondentes aos números marcados, sendo que a pontuação cumulativa pode variar de 0 a 128 , significando que quanto maior a pontuação, maior a percepção positiva do relacionamento com a pessoa. ${ }^{9,10}$

Os dados coletados foram sistematizados em planilha matriciada pelo estudo multicêntrico, diretamente no programa Statistical Package for the Social Sciencies (SPSS), versão 23.0, com dupla digitação e conferência. Foram realizadas análises descritivas, de tendência central (mediana e média) e de variabilidade (desvio-padrão), de acordo com cada variável (categórica ou contínua). A confiabilidade da escala utilizada foi avaliada pela consistência interna dos seus itens, medida pelo Coeficiente de alfa de Cronbach, sendo considerados com evidência de confiabilidade os valores acima de $0,70 .{ }^{11}$

O projeto de pesquisa foi aprovado pelo Comitê de Ética em Pesquisa da Escola de Enfermagem Anna Nery, sob parecer número 2.507.525 e CAAE: 80711517.8.1001.5238 em 22 de fevereiro de 2018.

\section{RESULTADOS}

Dentre as 152 participantes do estudo, a média de idade foi de 25,6 anos, com uma mediana de 25 anos, variando de 18 a 40 anos, dos quais eram predominantemente brancas $(51,7 \%)$, viviam com companheiro ou esposo $(84,9 \%)$, exerciam atividade laboral fora de casa $(54,6 \%)$ e não possuíam plano de saúde (80,9\%). Mencionaram, ainda, que a renda familiar era suficiente para as necessidades básicas (72,4\%); não fumavam (92,1\%) e não ingeriram bebida alcoólica nos últimos 3 meses (87,5\%), conforme Tabela 1.

Em relação à pessoa adulta mais importante, sobre a quem ela se referiu quanto às questões da escala $A R I$, o "companheiro ou esposo" foi a pessoa mais mencionada ( $58,6 \%)$, conforme Tabela2 2 .

Os itens da escala ARI com suas opções de respostas estão descritos na Tabela 3 , a seguir, com destaque para as respostas com maior frequência, assinaladas em negrito.

A avaliação da ARI está apresentada na Tabela 4, tanto os valores do total da escala (ARI total), bem como de suas duas dimensões (Apoio/Atitude positiva e Domínio/Controle).

Tabela 1. Caracterização sociodemográfica das participantes do estudo. Cascavel, PR, Brasil, 2020. $(n=152)$

\begin{tabular}{lcc}
\hline \multicolumn{1}{c}{ Variável } & n (\%) & (\%) \\
\hline Etnia & 78 & 51,7 \\
\hline Branca & 60 & 39,7 \\
\hline Parda & 10 & 6,6 \\
\hline Preta & 3 & 2,0 \\
\hline Amarela & & \\
\hline Estado civil & 129 & 84,9 \\
\hline Vivia com o companheiro ou esposo & 111 & 73,0 \\
\hline Casada & 83 & 54,6 \\
\hline Exerce atividade laboral fora de casa & 123 & 80,9 \\
\hline Plano de saúde (não) & 110 & 72,4 \\
\hline Renda familiar & 31 & 20,4 \\
\hline Suficiente para as necessidades básicas & 133 & 87,5 \\
\hline Mais do que o suficiente para as & 11 & 7,2 \\
\hline necessidades básicas & & \\
\hline $\begin{array}{l}\text { Menos do que o suficiente para as } \\
\text { necessidades básicas }\end{array}$ & 140 & 92,1 \\
\hline Não fumante & & \\
\hline Não ingeriu bebida alcoólica nos últimos & & \\
três meses & & \\
\hline
\end{tabular}

Fonte: Banco de dados da pesquisa.

Tabela 2. Pessoa adulta mais importante para a gestante, que atua como sua fonte de apoio primária. Cascavel, PR, Brasil, 2020. $(n=152)$

\begin{tabular}{lccc}
\hline \multicolumn{1}{c}{ Pessoa mencionada } & $\mathrm{n}$ & $\%$ \\
\hline Companheiro ou esposo & 89 & 58,6 \\
Outro membro da família & 61 & 40,1 \\
Outra pessoa não membro da família (amiga) & 2 & 1,3 \\
\hline
\end{tabular}

Fonte: Banco de dados da pesquisa. 
Tabela 3. Respostas da escala ARI. Cascavel, PR, Brasil, 2020. $(n=152)$

\begin{tabular}{|c|c|c|c|c|c|}
\hline Item da escala & $\begin{array}{c}\text { Não, } \\
\text { nunca(\%) }\end{array}$ & $\begin{array}{l}\text { Um pouco } \\
\text { (\%) }\end{array}$ & $\begin{array}{l}\text { Às vezes } \\
(\%)\end{array}$ & Muito (\%) & $\begin{array}{c}\text { Muitíssimo/ } \\
\text { Sempre } \\
(\%)\end{array}$ \\
\hline 1) Fala de seus problemas comigo & 3,3 & 9,9 & 13,8 & 19,7 & 53,3 \\
\hline 2) Sempre está tentando me mudar & 40,8 & 16,4 & 24,3 & 5,3 & 13,2 \\
\hline 3) Respeita minhas opiniões & 2,0 & 6,7 & 26,0 & 24,7 & 40,7 \\
\hline 4) Age como se eu o incomodasse & 80,3 & 11,2 & 7,2 & 1,3 & 0 \\
\hline 5) Está ali quando necessito dele(a) & 0,7 & 0,7 & 3,3 & 13,8 & 81,6 \\
\hline $\begin{array}{l}\text { 6) Não aceita um não como resposta quando ela/ele } \\
\text { necessita de algo }\end{array}$ & 56,3 & 11,9 & 23,2 & 2,6 & 6,0 \\
\hline 7) Tenta entender meu ponto de vista & 2,0 & 3,3 & 17,8 & 27,6 & 49,3 \\
\hline 8) Me dá toda a liberdade que quero & 5,9 & 4,6 & 21,1 & 22,4 & 46,1 \\
\hline 9) Está sempre pensando em coisas para me agradar & 2,6 & 3,3 & 14,5 & 25,0 & 54,6 \\
\hline 10) Discute, sem se importar com o que eu queira lhe dizer & 59,9 & 13,2 & 19,7 & 2,0 & 5,3 \\
\hline 11) Estimula-me a seguir meus próprios interesses & 2,6 & 4,6 & 14,6 & 18,5 & 59,6 \\
\hline 12) Ele (a) ri (zomba) de mim & 72,8 & 4,6 & 13,9 & 2,0 & 6,6 \\
\hline 13) Está muito disposto(a) a me ajudar quando necessito & 0 & 0,7 & 5,3 & 14,5 & 79,6 \\
\hline $\begin{array}{l}\text { 14) Quer ter a última palavra em como gastamos nosso } \\
\text { dinheiro }\end{array}$ & 63,8 & 11,2 & 16,4 & 2,0 & 6,6 \\
\hline 15) Pensa que vale a pena me escutar & 0,7 & 3,3 & 21,7 & 22,4 & 52,0 \\
\hline 16) Permite que eu mude de opinião & 4,6 & 0,7 & 30,3 & 21,1 & 43,4 \\
\hline 17) Passa um bom tempo comigo & 0,7 & 8,6 & 10,6 & 24,5 & 55,6 \\
\hline 18) Quer controlar tudo o que faço & 68,4 & 14,5 & 9,9 & 1,3 & 5,9 \\
\hline 19) Fica feliz em apoiar as minhas decisões & 3,3 & 2,6 & 16,4 & 23,0 & 54,6 \\
\hline 20) Diz que eu sou um problema para ele/ela & 91,4 & 3,9 & 2,6 & 0 & 2,0 \\
\hline $\begin{array}{l}\text { 21) Faz o possível para tornar as coisas mais fáceis para } \\
\text { mim }\end{array}$ & 0,7 & 2,0 & 7,9 & 21,1 & 68,4 \\
\hline 22) Espera que eu faça todas as coisas do seu jeito & 59,2 & 16,4 & 13,8 & 3,3 & 7,2 \\
\hline $\begin{array}{l}\text { 23) Faz com que eu sinta que posso lhe dizer o que eu } \\
\text { quiser }\end{array}$ & 4,6 & 6,6 & 17,1 & 15,8 & 55,9 \\
\hline $\begin{array}{l}\text { 24) Pensa que não tem problema algum não concordar com } \\
\text { ele/ela }\end{array}$ & 15,8 & 13,8 & 27,0 & 13,8 & 29,6 \\
\hline $\begin{array}{l}\text { 25) Me pede que compartilhe com ele/ela as coisas que } \\
\text { gosta }\end{array}$ & 3,9 & 2,6 & 11,2 & 21,1 & 61,2 \\
\hline 26) Sempre encontra defeitos em mim & 63,8 & 14,5 & 16,4 & 2,0 & 3,3 \\
\hline 27) Considera meu ponto de vista & 1,3 & 3,9 & 22,4 & 26,3 & 46,1 \\
\hline 28) Não pensa em mim & 77,6 & 5,3 & 3,3 & 5,9 & 7,9 \\
\hline 29) Trata de me consolar quando as coisas não vão bem & 3,9 & 0,7 & 4,6 & 23,0 & 67,8 \\
\hline $\begin{array}{l}\text { 30) Age como se não me conhecesse quando está } \\
\text { aborrecido }\end{array}$ & 77,5 & 7,9 & 7,9 & 3,3 & 3,3 \\
\hline $\begin{array}{l}\text { 31) Deseja que lhe conte quando algo está me } \\
\text { incomodando }\end{array}$ & 3,9 & 2,6 & 9,2 & 21,1 & 63,2 \\
\hline 32) Me deixa fazer qualquer coisa que eu queira fazer & 7,2 & 6,6 & 34,2 & 21,7 & 30,3 \\
\hline
\end{tabular}

Fonte: Banco de dados da pesquisa. 
Tabela 4. Distribuição dos escores da ARI total e suas dimensões. Cascavel, PR, Brasil, 2020. (n=152)

\begin{tabular}{lccccc}
\hline \multicolumn{1}{c}{ Itens } & No itens & Média \pm D.P. & Mediana & Variação & Alfa de Cronbach \\
\hline Apoio/ Atitude positiva & 20 & $58,6 \pm 9,9$ & 60,0 & 21 a 72 & 0,87 \\
Domínio/Controle & 12 & $37,9 \pm 7,3$ & 39,0 & 12 a 48 & 0,77 \\
ARI total & 32 & $103,5 \pm 15,9$ & 106 & 40 a 128 & 0,89 \\
\hline
\end{tabular}

D.P.= Desvio Padrão

Na dimensão apoio/atitude positiva, observou-se que mais de $50 \%$ das participantes apontaram que esse relacionamento apresenta aspectos positivos, como o suporte, escuta, aceitação e autonomia. Da mesma forma, quando mais de $70 \%$ das mulheres assinalaram a opção "Não/nunca" para as questões "age como se eu o incomodasse", "não pensa em mim" e "age como se não me conhecesse quando está aborrecido", tem-se o reforço de que a qualidade desse relacionamento é saudável.

No que concerne à dimensão Domínio/Controle da escala ARI, verificou-se que dos 12 itens, oito foram respondidos por mais de $50 \%$ das participantes com a opção "nunca", os quais foram: não aceita um não como resposta quando necessita de algo, discute, sem se importar com o que eu queira lhe dizer, ri (zomba) de mim, quer ter a última palavra em como gastamos nosso dinheiro, quer controlar tudo o que faço, diz que eu sou um problema para ele/ela, espera que eu faça todas as coisas do seu jeito e sempre encontra defeitos em mim. Essas respostas apontam um relacionamento baseado na aceitação, compreensão, respeito e desprendimento, reforçando o aspecto positivo do relacionamento.

Os demais itens não apresentaram respostas com porcentagens significativas, mas 40,7\% responderam "muitíssimo/sempre" para o item "respeita minhas opiniões" e $46,1 \%$ para "considera meu ponto de vista". No entanto, no item "me deixa fazer qualquer coisa que eu queira fazer", 34,2\% assinalaram a opção "às vezes", o que pode suscitar um entendimento dúbio da pergunta, por ser abrangente, cabendo interpretações diversas.

O uso de respostas para avaliar, de forma mediana, alguém ou alguma coisa por meio de um instrumento pode ser uma solução rápida e aparentemente segura de escolha, em que o indivíduo não se posiciona ao certo, não se compromete; ou ao contrário, expressa uma dúvida. Ainda, provavelmente, por ser o ponto que o respondente não tem que se esforçar para analisar e responder. ${ }^{12}$

Tratando-se do resultado das duas dimensões, verificou-se que os valores médios da dimensão Apoio/Atitude positiva foram de 58,6 pontos, variando de 21 a 72 e alfa de Cronbach de 0,87 . Já para a dimensão Domínio/Controle, a média foi de 37,9, variando de 12 a 48 pontos e alfa de Cronbach de 0,77 . No resultado da avaliação total da escala ARI, o escore apresentou uma média de 103,5, variando de 40 a 128 pontos e o valor de alfa de Cronbach de 0,89.

\section{DISCUSSÃO}

Similarmente a este estudo, pesquisa realizada com 100 mulheres hispânicas residentes nos Estados Unidos apontou que $57 \%$ do grupo identificou seu parceiro como a principal relação íntima primária, 32\% a mãe e 11\% outros familiares, incluindo o pai e irmã. ${ }^{10}$

O fato de quase a totalidade de as mulheres viverem com o companheiro ou esposo pode justificar essa principal indicação como a pessoa mais importante. Nesta vertente, o apoio do companheiro é apontado como a principal forma de participação durante o período gestacional e vai além de apenas suporte financeiro, uma vez que inclui o aporte emocional. ${ }^{13}$

A literatura aponta que relacionamentos amorosos saudáveis são baseados no respeito e na confiança, e sua qualidade está diretamente relacionada aos afetos positivos e à satisfação conjugal, podendo tornar-se importante fator de proteção de saúde mental. ${ }^{14}$ Como nesse estudo as mulheres têm a presença do companheiro, isso pode ser fator protetor para sua saúde mental, o que, por sua vez, poderia contribuir para sua confiança em condutas com o filho.

Ainda, neste contexto, sendo o companheiro/esposo mencionado frequentemente dentre as pessoas mais importantes para a mulher, pesquisa realizada na região noroeste do Paraná identificou que o parceiro/pai reconhece a importância de seu apoio para o sucesso da amamentação, principalmente em relação à sua presença junto à mãe e bebê, a ajuda nos afazeres domésticos, cuidados com o bebê e tentativas de amenizar as dificuldades vivenciadas durante a amamentação. ${ }^{15}$

Esses dados se assemelham às respostas da escala ARI, nas quais as mulheres afirmaram que a pessoa mencionada está sempre presente quando ela necessita, disposta a ajudá-la, a ficar com ela, fazendo o possível para tornar as coisas mais fáceis. Similarmente, estudo revela que o fato de as mulheres terem o acompanhamento do marido ou companheiro parece exercer influência positiva na duração do aleitamento. ${ }^{16}$

Em contrapartida, embora o cônjuge seja apontado como maior fonte de apoio em nosso estudo, autores identificaram que, quando ocorrem intercorrências mamárias, eles não oferecem esse suporte, ${ }^{17}$ porque não receberam as orientações durante o pré-natal sobre essa temática, que os ajudasse a compreender a situação e as dificuldades desse processo. ${ }^{15}$ Sendo assim, torna-se essencial que a atenção pré-natal esteja centrada na família, que a pessoa considerada mais importante para a mulher seja identificada e participe de todos os eventos realizados neste cenário. Dessa forma, torna-se necessário repensar as formas de atenção à saúde, estimular e facilitar a participação desses atores sociais no processo. ${ }^{13}$

Ainda nessa temática, entendendo o suporte social como o apoio emocional e instrumental, ${ }^{18}$ ao ser correlacionado o suporte social e relacionamento conjugal, evidenciou-se que quanto mais 
positiva era a relação entre os pares, expressada por carinho, comunicação entre o casal, coisas boas que o companheiro faz, entre outras características, maiores eram os suportes sociais disponíveis a eles, já que o relacionamento conjugal pode afetar as demais relações interpessoais e influenciar a procura e solicitação por suporte no seio familiar ou no contexto social. ${ }^{19}$ Assim, as respostas fornecidas pelas mulheres do estudo apontam para um relacionamento conjugal/familiar agradável com maiores disponibilidades para suportes sociais de apoio.

Tratando-se do resultado das duas dimensões, valores semelhantes foram encontrados no estudo de Linares, Hall e Ashford (2015), no qual o alfa de Cronbach foi de 0,91 para a dimensão Apoio/Atitude positiva e 0,82 para a dimensão Domínio/ Controle. Para a escala total a média foi de 108,8 , variando de 59 a 128; e o valor de alfa de Cronbach de 0,92, indicando sua solidez para medir a qualidade de uma relação íntima primária naquela amostra. ${ }^{10}$

Os valores descritivos da escala ARI demonstraram que, predominantemente, as mulheres possuíam relação positiva com sua fonte de apoio primária. Pesquisa realizada com a finalidade de identificar a percepção de gestantes acerca do apoio familiar recebido durante o período gravídico-puerperal, demonstrou que a maioria das entrevistadas possuíam uma percepção positiva sobre o apoio recebido e valorizavam o envolvimento familiar nesse período, o que influencia positivamente o período prénatal, parto e puerpério, incluindo nesse a amamentação. ${ }^{16}$ Ao se relacionarem com sua rede social durante a amamentação, as nutrizes esperam receber compreensão e apoio e apontam a intenção de manter essas relações com um aspecto positivo para superarem as dificuldades e obterem sucesso na amamentação. ${ }^{20}$ Uma relação positiva com uma pessoa considerada como fonte de apoio primária pode influenciar positivamente a iniciação e manutenção do AM.

Para tanto, utilizar ferramentas que auxiliem na identificação do apoio primário para a gestante na atenção primária à saúde possibilita à equipe de saúde direcionar suas ações com enfoque no cuidado para além da mulher, considerando seus vínculos estabelecidos. Nesta investigação, a escala ARI mostrou-se método efetivo para avaliar a qualidade de um relacionamento com uma pessoa próxima, pois quando seus resultados são positivos, maior é o apoio recebido pela gestante e, consequentemente, maiores são as influências para que a mulher inicie e mantenha - AME. Ao contrário, quando o resultado é negativo, a equipe de saúde pode dedicar-se a construir esse relacionamento na família para o suporte que a mulher irá necessitar durante a gestação e após o nascimento do bebê.

\section{CONCLUSÃO E IMPLICAÇÕES PARA A PRÁTICA}

Os resultados dessa pesquisa demonstraram que as gestantes identificam o companheiro/esposo e outros membros da família como sendo as pessoas mais importantes e que atuam como sua fonte de apoio primária, demonstrando a necessidade de desenvolver a atenção à saúde centrada na família. Esse não é um tema novo para a Enfermagem, tampouco para a atenção primária, contudo, continua na pauta dos temas pendentes, relacionada com a qualidade do pré-natal no país, impactando os números da mortalidade materno-infantil.

Nos resultados da escala ARI, verificou-se que a qualidade do relacionamento com a pessoa próxima é saudável e possui aspectos positivos, uma vez que muitas questões abordadas que envolviam características de suporte, escuta, autonomia, aceitação, compreensão e respeito foram respondidas positivamente. Sugere-se sua utilização nas unidades de atenção primária para a identificação do apoio primário das gestantes e atuação do Enfermeiro no fortalecimento dos vínculos para o suporte necessário à mulher no período gravídico-puerperal.

O estudo apresenta limitações como a amostra não aleatorizada, a não inclusão de outras avaliações, como da equipe de saúde e a ausência de instrumentos para avaliação do apoio à gestante em língua portuguesa. Por esse motivo, a comparação com os demais instrumentos utilizados para avaliação do aleitamento materno na gestação inviabilizou outros tipos de análise de correlação entre o apoio recebido e o AME.

\section{CONTRIBUIÇÕES DOS AUTORES}

Desenho do estudo. Janaine Fragnan Peres. Cláudia Silveira Viera. Ana Maria Linares. Marialda Moreira Christoffel. Beatriz Rosana Gonçalves de Oliveira Toso.

Coleta ou produção dos dados. Janaine Fragnan Peres. Cláudia Silveira Viera. Beatriz Rosana Gonçalves de Oliveira Toso.

Análise de dados e interpretação dos resultados. Janaine Fragnan Peres. Ariana Rodrigues Silva Carvalho. Beatriz Rosana Gonçalves de Oliveira Toso.

Redação e revisão crítica do manuscrito. Janaine Fragnan Peres. Ariana Rodrigues Silva Carvalho. Cláudia Silveira Viera. Ana Maria Linares. Marialda Moreira Christoffel. Beatriz Rosana Gonçalves de Oliveira Toso.

Aprovação da versão final do artigo. Janaine Fragnan Peres. Ariana Rodrigues Silva Carvalho. Cláudia Silveira Viera. Ana Maria Linares. Marialda Moreira Christoffel. Beatriz Rosana Gonçalves de Oliveira Toso.

Responsabilidade por todos os aspectos do conteúdo e a integridade do artigo publicado. Janaine Fragnan Peres. Ariana Rodrigues Silva Carvalho. Cláudia Silveira Viera. Ana Maria Linares. Marialda Moreira Christoffel. Beatriz Rosana Gonçalves de Oliveira Toso.

\section{EDITOR ASSOCIADO}

Stela Maris de Mello Padoin

\section{REFERÊNCIAS}

1. Ministério da Saúde (BR), Secretaria de Atenção à Saúde, Departamento de Atenção Básica. Saúde da Criança: aleitamento materno e alimentação complementar [Internet]. Brasília (DF): Ministério da Saúde; 2015 
[citado 2020 abr 29]. Disponível em: https://bvsms.saude.gov.br/bvs/ publicacoes/saude_crianca_aleitamento_materno_cab23.pdf

2. Rollins NC, Bhandari N, Hajeebhoy N, Horton S, Lutter CK, Martines JC, et al. Why invest, and what it will take to improve breastfeeding practices? Lancet. 2016; jan;387(10017):491-504. http://dx.doi. org/10.1016/S0140-6736(15)01044-2.

3. Boccolini CS, Carvalho ML, Oliveira MI. Factors associated with exclusive breastfeeding in the first six months of life in Brazil: a systematic review. Rev Saude Publica. 2015;49:91. http://dx.doi.org/10.1590/S00348910.2015049005971. PMid:26759970.

4. Prates LA, Schmalfuss JM, Lipinski JM. Rede de apoio social de puérperas na prática da amamentação. Esc Anna Nery. 2015;19(2):310-5. http:// dx.doi.org/10.5935/1414-8145.20150042.

5. Cardoso ACA, Vivian AG. Maternidade e suas vicissitudes: a importância do apoio social no desenvolvimento da díade mãe-bebê. Diaphora. [Internet]; 2017 [citado 2020 abr 24];6(1):43-51. Disponível em: http:// www.sprgs.org.br/diaphora/ojs/index.php/diaphora/article/view/134/139

6. Primo CC, Dutra PR, Lima EFA, Alvarenga SC, Leite FMC. Redes sociais que apoiam a mulher durante a amamentação. Cogitare Enferm. 2015;20(2):426-33. http://dx.doi.org/10.5380/ce.v20i2.37453.

7. Amaral LJX, Sales SS, Carvalho DPSRP, Cruz GKP, Azevedo IC, Ferreira Jr MA. Fatores que influenciam na interrupção do aleitamento materno exclusivo em nutrizes. Rev Gaúcha Enferm. 2015;36(N. esp.):127-34. http://dx.doi.org/10.1590/1983-1447.2015.esp.56676.

8. Riemenschneider PS, D’Aquino L. Maternidade, redes sociais e sociedade de consumo: vulnerabilidade ou empoderamento da lactante? Anais do $4^{\circ}$ Congresso Internacional de Direito e Contemporaneidade [Internet]; 2017; Santa Maria (RS), Brasil. Santa Maria (RS): UFSM; 2017. p. 1-14 [citado 2020 abr 24]. Disponível em: http://coral.ufsm.br/ congressodireito/anais/2017/5-2.pdf

9. Christoffel MM, Rodrigues EC, Araujo LSC, Gomes ALM, Machado MED, Toso BRGO, et al. Tradução, adaptação e validação da Escala Calidad de la relación con su persona cercana. Rev Rene. 2020;21:e44029. http://dx.doi.org/10.15253/2175-6783.20202144029.

10. Linares AM, Hall L, Ashford K. Psychometric testing of the Autonomy and Relatedness Inventory - Spanish Version. J Nurs Meas. 2015;23(1):E2737. http://dx. doi.org/10.1891/1061-3749.23.1.E27. PMid:26269139.
11. Fayers PM, Machin D. Scores and measurements: validity, reliability, sensitivity. In: Fayers PM, Machin D. Quality of life: the assessment, analysis and interpretation of patient-reported outcomes. 2nd ed. New York: Wiley; 2007. p. 77-108. http://dx.doi.org/10.1002/9780470024522.ch4.

12. Costa FJ, Orsini ACR, Carneiro JS. Variações de mensuração por tipos de escalas de verificação: uma análise do construto de satisfação discente. Rev GestãoOrg. 2018;16(2):132-44. http://dx.doi.org/10.21714/167918272018v16n2.p132-144.

13. Caldeira LA, Ayres LFA, Oliveira LVA, Henriques BD. A visão das gestantes acerca da participação do homem no processo gestacional. Rev Enferm do centro-oeste Min. 2017; 7(e1417):1-10. https://doi. org/10.19175/recom.v7i0.1417.

14. Almeida Costa NB, Modesto JG. Representação social do relacionamento amoroso saudável social. Rev Psicol da IMED. 2020;12(1):100-15. http://dx.doi.org/10.18256/2175-5027.2020.v12i1.3497.

15. Teston EF, Reis TS, Góis LM, Spigolon DN, Maran E, Marcon SS. Aleitamento materno: percepção do pai sobre seu papel. Rev Enferm do centro-oeste Min. 2018;8(e2723):1-7. http://dx.doi.org/10.19175/recom.v8i0.2723.

16. Avanzi SA, Dias CA, Silva LOL, Brandão MBF, Rodrigues SM. Importância do apoio familiar no período gravídico-gestacional sob a perspectiva de gestantes inseridas no PHPN. Rev Saúde Col UEFS. 2019;9:55-62. http://dx.doi.org/10.13102/rscdauefs.v9i0.3739.

17. Silva FA, Cantanhede NAC, Fonseca PCA, Conceição SIO. Apoio social e intercorrências mamárias de nutrizes que amamentam exclusivamente. DEMETRA - Aliment Nutr Saúde. 2019;14(Supl 1):1-16. https://doi. org/10.12957/demetra.2019.43824.

18. Leitao Maia CM, Vicente Castro F, Godinho da Fonseca AM, Ruiz Fernández MI. Redes de apoio social e de suporte social e envelhecimento ativo. Rev INFAD Psicol. 2016;1(1):293-304. http://dx.doi.org/10.17060/ ijodaep.2016.n1.v1.279.

19. Azevedo TL, Cia F, Spinazola CC. Correlação entre o relacionamento conjugal, rotina familiar, suporte social, necessidades e qualidade de vida de pais e mães de crianças com deficiência. Rev Bras Educ Espec. 2019;25(2):205-18. http://dx.doi.org/10.1590/s1413-65382519000200002.

20. Souza MHN, Nespoli A, Zeitoune RCG. Influência da rede social no processo de amamentação: um estudo fenomenológico. Esc Anna Nery. 2016;20(4):1-6. http://dx.doi.org/10.5935/1414-8145.20160107. 